[4] M. Krzyżański et A. Szybiak, Construction et étude de la solution fondamentale de l'équation linéaire du type parabolique dont le dernier coefficient est non borné, Note I e II, Atti Accad. Naz. Lincei, el. Sc. fis., mat. e natur., ser. VIII, 27 (1959), p. 1-10.

[5] A. Szybiak, On the Asymptotic Behaviour of the Solutions of the Equation Ond 7 (1959) p. 183-186.

\section{Sur l'allure asymptotique des solutions de l'équation différentielle ordinaire du second ordre}

par T. D£omko (Katowice)

Il existe une classe d'équations différentielles ordinaires du second ordre de la forme

$$
u^{\prime \prime}(t)+g(t, u(t))=0,
$$

possédant de nombreuses propriétés de l'équation différentielle linéaire $u^{\prime \prime}(t)+a(t) u(t)=0$.

Je vais démontrer dans cette note les propriétés que voici: les solutions de l'équation (1) existent dans tout l'intervalle $\left\langle t_{0}, \infty\right.$ ), elles sont bornées, oscillantes ou non oscillantes, elles possèdent des asymptotes, les suites de leurs extrêmes sont monotones, etc.

En particulier, dans le cas où $g(t, u)=a(t) f(u)$, je généralise le résultats obtenus à l'équation différentielle $\left(k(t) u^{\prime}\right)^{\prime}+l(t) / k(t) f(u)=0$.

En outre, les théorèmes demontrés entraînent, comme propositions, certains théorèmes établis par les auteurs [1], [2], [7], [8], [9], [12], [13], [15], [16].

Le trait caractéristique de cette note sera l'emploi de simples et brèves méthodes de démonstration.

J'ai l'honneur de remercier M. A. Bielecki pour ses précieuses remaiques et suggestions, et M. Z. Opial pour son aide dans la rédaction de cette note.

I. Dans cette partie de la note nous allons démontrer un théorème qui, entre autres, assure l'existence des solutions de l'équation (1) dans l'intervalle $t \geqslant t_{0}$.

THस́ов亡̀ME 1. Considérons le système d'équations différentielles

$$
x_{i}^{\prime}(t)=f_{i}\left(t, x_{1}(t), \ldots, x_{n}(t)\right), \quad i=1, \ldots, n,
$$

dans lequel les fonctions $f_{i}$ sont définies et continues dans le domaine $D\left\{t \geqslant t_{0},-\infty<x_{i}<+\infty, i=1, \ldots, n\right\}$. Nous supposons qu'il existe une fonction $w(t, u)$ définie, continue et non négative dans le domaine $D_{1}\left\{t \geqslant t_{0}, u \geqslant 0\right\}$ et telle que l'équation différentielle

$$
u^{\prime}(t)=w(t, u(t)),
$$

ait toutes ses solutions $u(t)$ définies dans tout l'intervalle $t \geqslant t_{0}$. 
si $\|f(t, x)\| \leqslant w(t,\|x\|)$, ò̀ $x(t)=\left\{x_{1}(t), \ldots, x_{n}(t)\right\}$ désigne un vecteur dont la norme est $\|x(t)\|$ chaque solution du système (2), satisfaisant aux conditions initiales $x_{i}\left(t_{0}\right)=x_{i 0}, i=1, \ldots, n$, définie dans l'intervalle $\left\langle t_{0}, \beta\right)$, $\beta<+\infty$, peut être prolongée sur tout l'intervalle $t \geqslant t_{0}$.

Démonstration. En vertu du théorème classique sur l'existence des solutions du système d'équations différentielles (2), il existe une solution de ce système, vérifiant les conditions initiales, définie dans le plus grand intervalle $\left\langle t_{0}, \beta\right)$, où $\beta>t_{0}$. Nous allons démontrer que l'hypothèse $\beta<+\infty$ conduit à l'absurde.

On sait que lorsque les fonctions $f_{i}$ sont continues dans le domaine $D$ et la solution $x_{i}\left(t, t_{0}, x_{10}, \ldots, x_{n 0}\right)$ existe seulement dans le plus grand intervalle $\left\langle t_{0}, \beta\right)$ et $\beta<+\infty$, alors $\left\|x\left(t, t_{0}, \ldots, x_{n_{0}}\right)\right\|$ tend vers $+\infty$, quand $t \rightarrow \beta-0 \quad([3]$, p. 3$)$.

Les fonctions $x_{i}\left(t, t_{0}, \ldots, x_{n_{0}}\right) \quad\left(t_{0} \leqslant t<\beta\right)$ sont continues dans cet intervalle. Il s'ensuit que la fonction $\left\|x\left(t, t_{0}, \ldots, x_{n 0}\right)\right\|$ est continue quand $t_{0} \leqslant t<\beta$. Choisissons un point $\left(t_{0}, u_{0}\right)$ tel que $u_{0}>\left\|x_{i 0}\right\|, i=1, \ldots, n$. Désignons par $\varphi(t)$ l'intégrale supérieure de l'équation (3), définie par la condition initiale $\left(t_{0}, u_{0}\right)$. Par hypothèse, la fonction $\varphi(t)$ est définie pour $t \geqslant t_{0}$. Il existe donc un dernier point $\tau$ tel que $\left\|x\left(t, t_{0}, \ldots, x_{n_{0}}\right)\right\|$ $=\varphi(\tau)$ et

$$
\left\|x\left(t, t_{0}, \ldots, x_{n 0}\right)\right\|>\varphi(t) \quad \text { pour } \quad t>\tau .
$$

On peut démontrer la formule $D\|x(t)\| \leqslant\left\|x^{\prime}(t)\right\|$, où $D\|x(t)\|$ désigne un nombre dérivé arbitraire de la fonction continue $\left\|x\left(t, t_{0}, \ldots, x_{n_{0}}\right)\right\|$ pour $t \geqslant t_{0}$. En particulier $D_{+}\|x(t)\|$ et $n_{-}\|x(t)\|$ ne surpassent pas $\left\|x^{\prime}(t)\right\|$. Ainsi donc, d'après les hypothèses admises,

$$
\begin{array}{lll}
\underline{D}_{+}\|x(t)\| \leqslant w(t,\|x(t)\|) & \text { pour } & t>\tau, \\
\underline{D}_{-}\|x(t)\| \leqslant w(t,\|x(t)\|) & \text { pour } & t>\tau .
\end{array}
$$

En vertu de [14], p. 124, nous avons

$$
\left(3^{\prime \prime}\right) \quad\left\|x\left(t, t_{0}, \ldots, x_{n_{0}}\right)\right\| \leqslant \varphi(t) \text { pour } \quad t>\tau .
$$

Les relations $\left(3^{\prime}\right)$ et $\left(3^{\prime \prime}\right)$ sont contradictoires, on ne peut done pas avoir $\beta<+\infty$, ce qui démontre la conclusion du théorème.

Remarque 1 . Si, dans le théorème 1 , nous admettions encore que la fonction $w(t, u)$ était croissante par rapport à la variable $u(u \geqslant 0)$, nous pourrions obtenir une condition suffisante pour que toutes les solutions du système (2) existent dans tout l'intervalle $\left\langle t_{0}, \infty\right)$. Ce théorème a été démontré par $\mathrm{A}$. Bielecki et cité par J. Kisyński [7].

Remarque 2. Si l'on admet $w(t, u)=A(t) u+B(t) \quad(A(t) \geqslant 0$, $B(t) \geqslant 0$ pour $\left.t \geqslant t_{0}\right)$, on déduit du théorème 1 une condition suffisante pour que toutes les solutions du système (2) existent dans l'intervalle $\left\langle t_{0}, \infty\right)$. Cette condition a été démontrée par $\mathbf{A}$. Wintner [15].
Nous énoncerons maintenant quelques hypothèses qui interviendront dans nos théorèmes.

HYPOTHEेSE $\mathrm{H}_{1}$. La fonction $a(t)>0$ est définie et continue et possède une dérivée $a^{\prime}(t)$ continue pour $t \epsilon\langle 0, \alpha)$. La fonction $a^{\prime}(t)$ satisfait pour $t \epsilon\langle 0, \alpha)$ à l'une au moins des conditions:

$1^{\circ} \quad a^{\prime}(t) \geqslant 0$, ou

$2^{\circ} a(t) \geqslant \delta>0$ et $\int_{0}^{a}\left|a^{\prime}(t)\right| d t<\infty$.

La fonction $f(u)$ est définie et continue pour $u \in(-\infty,+\infty)$ et telle que

$$
F(u)=\int_{0}^{u} f(x) d x \rightarrow+\infty, \quad \text { pour } \quad|u| \rightarrow+\infty .
$$

Dans l'hypothèse $\mathrm{H}_{1}$, la fonction $F(u)$ est continue et tend vers $+\infty$, quand $u \rightarrow+\infty$ ou bien $u \rightarrow-\infty$. Il en résulte l'existence de $\min F(u)=F(p)$ $=-\mu \leqslant 0$ et $G(u)=F(u)+\mu=\int_{p}^{u} f(x) d x \geqslant 0$, pour $u \in(-\infty,+\infty)$. Considérons l'équation différentielle

$$
u^{\prime \prime}(t)+a(t) f(u)=0,
$$

dans laquelle les fonctions $a(t)$ et $f(u)$ satisfont à l'hypothèse $\mathrm{H}_{1}$. Soit $u(t)$ une solution de l'équation (4), déterminée par les conditions initiales $u(0)=u_{0}, u^{\prime}(0)=v_{0}$. Multiplions l'équation (4) par $2 u^{\prime}(t)$ et intégrons dans l'intervalle $\langle 0, t\rangle$, dans lequel cette solution existe. Nous aurons l'égalité

$\left(4^{\prime}\right) \quad\left(u^{\prime}(t)\right)^{2}+2 a(t) G(u(t))=v_{0}^{2}+2 a(0) G\left(u_{0}\right)+2 \int_{0}^{t} a^{\prime}(\tau) G(u(\tau)) d \tau$, d'où

(5) $a(t) G(u(t)) \leqslant \frac{C}{2}+\int_{0}^{t} \frac{\left|a^{\prime}(\tau)\right|}{a(\tau)} G(u(\tau)) a(\tau) d \tau$, où $C=v_{0}^{2}+2 a(0) G\left(u_{0}\right)$,

$$
\left(\left.u^{\prime}(t)\right|^{2} \leqslant C+2 \int_{0}^{t}\left|a^{\prime}(\tau)\right| G(u(\tau)) d \tau .\right.
$$

Nous pouvons appliquer à l'inégalité (5) le lemme de Bellman-Niemyckij ([10], p. 19)

$$
G(u(t)) \leqslant \frac{C}{2 a(t)} \exp \left(\int_{0}^{t} \frac{\left|a^{\prime}(\tau)\right|}{a(\tau)} d \tau\right) .
$$

En vertu des inégalités $\left(5^{\prime}\right)$ et $(6)$ nous pouvons formuler le théorème suivant. 
THÉoRغ̀ne 2. Considérons l'équation différentielle (4) et admettons l'hypothèse $\mathrm{H}_{1}$. Alors chaque solution $u(t)$ existe dans tout l'intervalle $\langle 0, \alpha)$. Cette solution est bornée, ainsi que sa dérivée $u^{\prime}(t)$, dans l'intervalle $\langle 0, \alpha)$.

Remarque 1. De l'inégalité $\left(5^{\prime}\right)$ nous tirons pour $t \in\langle 0, \alpha)$ l'inégalité $G(u(t)) \leqslant \mathrm{const}=c_{1}$, d'où $-\mu \leqslant F^{\prime}(u) \leqslant c_{1}-\mu$. Puisque $\lim F^{\prime}(u)$ $=+\infty$ quand $|u| \rightarrow+\infty$, il existe done deux nombres $u_{1}$ et $u_{2}$ tels que $F\left(u_{1}\right)=c_{1}-\mu$ et $F(u)>c_{1}-\mu$ pour $u<u_{1}$, et $F\left(u_{2}\right)=c_{1}-\mu$ et $F^{\prime}(u)$ $>c_{1}-\mu$ pour $u>u_{2}$. Ainsi, pour l'intégrale $u(t)$, satisfaisant aux conditions initiales $u(0)=u_{0}, u^{\prime}(0)=v_{0}$, on a l'inégalité $u_{1} \leqslant u(t) \leqslant u_{2}$ pour $t \in\langle 0, a)$.

Remarque 2. Si l'on admet daus le théorème $(2) a^{\prime}(t) \geqslant 0$, alors̀ on obtient un théorème établi par J. A. Klokoff ([8], p. 190). Des résultats plus généraux ont été donnés dans la note [6].

Passons maintenant à l'équation différentielle

$$
\left(k(t) u^{\prime}\right)^{\prime}+l(t) / k(t) f(u)=0 .
$$

Nous admettons que les fonctions $k(t)$ et $l(t)$ sont positives et continues dans l'intervalle $\langle 0, \infty)$. La transformation

$$
s(t)=\int_{0}^{t} \frac{1}{k(\tau)} d \tau \text { poụ } \quad t \in\langle 0, \infty)
$$

change l'équation (7) en

$$
v^{\prime \prime}(s)+l(t(s)) f(v(s))=0,
$$

où $t(s)$ est la fonction inverse de $s(t)$. La solution de cette dernière équatiou est la fonction $v(s)=u(t(s))$ si, et seulement si, la fonction $u(t)$ est solution de l'équation (7). La fonction $s(t)$ est positive et croissante pour $t \epsilon\langle 0, \infty)$. L'ensemble des valeurs de la fonction $s(t)$ constitue un intervalle $\langle 0, \beta)$, où

$$
\beta=\int_{0}^{\infty} \frac{1}{k(t)} d t
$$

Si, pour $t \geqslant 0, u(t)$ est intégrale de l'équation (7), alors $v(s)=u(t(s))$ pour $0 \leqslant s<\beta$ est intégrale de l'équation (8) et inversement. L'ensemble des valeurs de la fonction $u(t)$ pour $t \epsilon\langle 0, \infty)$ est identique à celui des valeurs de la fonction $v(s)=u(t(s))$ pour $s \in\langle 0, \beta)$. Il en résulte que les ensembles des valeurs des fonctions $F(u(t))$ pour $t \epsilon\langle 0, \infty)$ et $F(v(s)$ pour $s \epsilon\langle 0, \beta\rangle$ sont identiques. On peut observer que, si $u(t)$ est une fonction croissante (décroissante), alors la fonction correspondante $v(s)$ est croissante (décroissante), puisque la fonction $s(t)$ est croissante.
HYPOTHĖSE $\mathrm{H}_{1}^{*}$. Les fonctions $k(t)$ et $l(t)$ sont positives et continues dans l'intervalle $\langle 0, \infty)$; la dérivée $l^{\prime}(t)$ est continue dans l'intervalle $\langle 0, \infty)$ et satisfait à l'une au moins des conditions:

$1^{0} l^{\prime}(t) \geqslant 0$ pour $t \in\langle 0, \infty)$

$2^{\circ} l(t) \geqslant \delta>0$ et $\int_{0}^{\infty}\left|l^{\prime}(t)\right| k(t) d t<+\infty$ pour $t \epsilon\langle 0, \infty)$.

La fonction $f(u)$ satisfait aux mêmes conditions que dans l'hypothèse $H_{1}$.

THEORÈme 3. Admettons que les fonctions $k(t), l(t)$ et $f(u)$ satisfassent à l'hypothèse $\mathrm{H}_{1}^{*}$. Alors chaque solution $u(t)$ de l'équation différentielle (7) existe dans tout l'intervalle $\langle 0, \infty)$. Cette solution est bornée, ainsi que sa dérivée $u^{\prime}(t)$, dans l'intervalle $\langle 0, \infty)$.

La démonstration de ce théorème est basée sur les propriétés de transformation de $\left(7^{\prime}\right)$ et sur le théorème 2 .

II. Dans la seconde partie de cette note, je me propose de donner une condition suffisante pour que toutes les solutions de l'équation différentielle (1) soient non oscillantes.

HYPoтHèse $\mathrm{H}_{2}$. La fonction $g(t, u)$ est définie et continue dans le domaine $D_{2}\left\{t \geqslant t_{0},-\infty<u<+\infty\right\}$ et elle y satisfait à la condition $g(t, u) u>0$ pour $u \neq 0$.

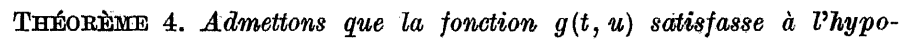
thèse $\mathrm{H}_{2}$. Alors chaque solution $u(t)$ de l'équation différentielle

$$
u^{\prime \prime}(t)-g(t, u(t))=0,
$$

ne $s^{\prime}$ annulant pas $\left.{ }^{1}\right)$ dans un intervalle tout entier; définie pour $t \geqslant t_{0}$, possède tout au plus un zéro (exactement: le produit $u(t) u^{\prime}(t) s^{\prime}$ annule une fois tout au plus pour $\left.t \geqslant t_{0}\right)$.

Démonstration. Soit $u(t)$ une solution de l'équation (9) telle que nous avons considérée plus haut. Prenons la fonction $\varphi(t)=u(t) u^{\prime}(t)$. Alors $\varphi^{\prime}(t)=g(t, u(t)) u(t)+\left(u^{\prime}(t)\right)^{2} \geqslant 0$. Cela veut dire que le produit $u(t) u^{\prime}(t)$ est une fonction non décroissante, il peut donc admettre tout au plus un zéro. Les solutions de l'équation (9) sont donc, au moins à partir d'un certain point, monotones.

Remarque. On peut aussi appliquer le théorème précédent à l'équation différentielle

$$
\left(k(t) u^{\prime}\right)^{\prime}-l(t) / k(t) f(u)=0
$$

dans laquelle les fonctions $k(t)>0$ et $l(t) \geqslant 0$ sont contimues pour $t \geqslant t_{0}$, et la fonction $f(u)$ est continue pour $u \in(-\infty,+\infty)$ et satisfait à la con-

(1) $\mathrm{Au}$ lieu de supposer que les' solutions ne s'annulent pas dans un certain intervalle, on peut admettre la condition d'unicité des solutions pour l'équation différentielle (9). 
dition $f(u) u>0$ pour $u \neq 0$. Cela résulte des propriétés de transformation de $\left(7^{\prime}\right)$ et du théorème précédent.

La dernière remarque généralise la condition connue de non oscillation des solutions de l'équation différentielle

$$
u^{\prime \prime}+a(t) u^{\prime}+b(t) u=0
$$

(voir [12], vol I, chap. IV, § 2) qu'on obtient de l'équation (10) en posant $f(u)=u$.

III. Dans cette partie, nous étudions l'oscillation de toutes les solutions de l'équation différentielle ( 1 ) dans l'intervalle $t \geqslant t_{0}$.

HYPoThìse $\mathrm{H}_{3}$. La fonction $g(t, u)$ satisfait à l'hypothèse $\mathrm{H}_{2}$ et on a en outre $\int^{\infty} g(t, v(t)) d t=+\infty(-\infty)$, où $v(t)>0(<0)$ est une fonction arbitraire, définie et continue pour $t \geqslant t_{0}$ et $v(t)$ est non décroissante (non croissante) dans cet intervalle.

Remarque. L'hypothèse $\mathrm{H}_{3}$ est vérifiée, par exemple, quand $g(t, u)$ $=a(t) f(u)\left(a(t)>0\right.$ pour $t \geqslant t_{0}$ et $f(u) u>0$ pour $\left.u \neq 0\right)$ et ces fonctions satisfont au moins à l'une des conditions
a) $\int^{\infty} a(t) d t=+\infty$ et $\liminf |f(u)| \geqslant \varepsilon_{0}>0$ pour $|u| \rightarrow+\infty$,
b) $a(t) \geqslant \delta>0$ et $\lim _{|u| \rightarrow \infty} F^{\prime}(u)=+\infty$, où $F(u)=\int_{0}^{u} f(x) d x$.

THÉم̇̀me 5. Admettons que la fonction $g(t, u)$ satisfasse à l'hypothèse $\mathrm{H}_{3}$. Alors chaque solution $u(t)$ de l'équation (1), définie pour $t \geqslant t_{0}$ oscille autour de la droite $u=0$, et l'ensemble des zéros n'est pas borné.

Démonstration. Admettons qu'il existe une solution $u(t)>0$ $(<0)$ pour $t \geqslant t_{0}$. Donc $u^{\prime}(t)$ n'est pas croissante pour $t \geqslant t_{1}$. Si l'on avait $u^{\prime}(t) \geqslant 0$ pour $t \geqslant t_{1}$ alors

$$
u^{\prime}(t)=u^{\prime}\left(t_{1}\right)-\int_{t_{1}}^{t} g(\tau, u(\tau)) d \tau,
$$

d'où, en vertu des propriétés de la fonction $g(t, u)$ nous aurions lim $u^{\prime}(t)$ $=-\infty$ quand $t \rightarrow+\infty$. Mais $u(t)>0$ pour $t \geqslant t_{0}$, donc il est impossible que $\lim u^{\prime}(t)=-\infty$ quand $t \rightarrow+\infty$. Il existe un tel $t_{2} \geqslant t_{1}$, que $u^{\prime}\left(t_{2}\right)<0$. De l'égalité

$$
u^{\prime}(t)=u^{\prime}\left(t_{2}\right)-\int_{t_{2}}^{t} g(\tau, u(\tau)) d \tau,
$$

nous déduisons que $u^{\prime}(t)$ pour $t \geqslant t_{2}$ est négative et décroissante, donc la fonction $u(t)$ derrait s'annuler, ce qui est absurde.

La contradiction achève la démonstration du théorème.
Remarque 1. Pour que le théorème précédent soit viai, il ne suffit pas que $g(t, u) u>0$ pour $u \neq 0$. La condition $\int^{\infty} g(t, v(t)) d t=+\infty(-\infty)$ est essentielle, parce que l'équation différentielle $u^{\prime \prime}+\frac{1}{4}\left(1+\left(u^{2}-t\right)^{2}\right) u^{1 / 8} t^{-5 / 2}$ $=0(t>0, u$ arbitraire $)$ satisfait à la condition $g(t, u) u>0$ pour $u \neq 0$.

Si l'on admet $v(t)=t^{1 / 2}$, alors $\int^{\infty} g\left(t, t^{1 / 2}\right) d t<+\infty$, la dernière équation possède une solution non oscillante $u(t)=t^{1 / 2}$.

Remarque 2. Du dernier théorème résulte aussi le corollaire suivant (voir les propriétés de transformation de $\left(7^{\prime}\right)$ ): Admettons que les fonctions $k(t)$ et $l(t)$ soient positives et continues pour $t \geqslant t_{0}, \int^{\infty} 1 / k(t) d t=+\infty$. La fonction $f(u)$ est définie et continue pour $u \in(-\infty,+\infty)$, et $f(u) u>0$ pour $u \neq 0$. Si l'une au moins des deux conditions

$$
\begin{aligned}
& \text { a) } \int^{\infty} l(t) / k(t) d t=+\infty \text { et } \liminf _{|u| \rightarrow \infty}|f(u)| \geqslant \varepsilon_{0}>0, \\
& \text { b) } l(t) / k(t) \geqslant \delta>0 \text { et } \lim _{|u| \rightarrow \infty} F(u)=+\infty \text { quand } F(u)=\int_{0}^{u} f(x) d x,
\end{aligned}
$$

est vraie, alors chaque solution $u(t)$ de l'équation différentielle (7), définie pour $t \geqslant t_{0}$, oscille autour de $u=0$ et possède un ensemble des zéros non borné supérieurement. Le dernier théorème généralise dans un certain sens le théorème connu relatif à l'équation différentielle linéaire ([13], p. 254).

IV. Nous passons maintenant à la démonstration de quelques théorèmes sur les solutions monotones de l'équation différentielle (7) et sur les limites finies de ces solutions.

THÉoRł̀me 6. Considérons l'équation différentielle (7) dans laquelle la fonction $k(t)$ est positive et continue dans l'intervalle $\langle 0, \infty)$. Admettons de plus que $\beta=\int^{\infty} 1 / k(t) d t<+\infty$ et que $\lim l(t)=l$ existe quand $t \rightarrow+\infty$ et $0<l<+\infty$. Si l'on peut appliquer le théorème 2 , à l'équation différentielle

(12)

$$
v^{\prime \prime}(s)+\gamma(s) f(v(s))=0,
$$

dans laquelle

$$
\gamma(s)=\left\{\begin{array}{lll}
l(t(s)) & \text { pour } & s \in<0, \beta), \\
l & \text { pour } & s \geqslant \beta,
\end{array}\right.
$$

alors l'intégrale $u(t)$ de l'équation (7), définie dans l'intervalle $t \geqslant 0$, tend vers une constante, quand $t \rightarrow+\infty$.

Démonstration. En vertu des hypothèses admises, l'intégrale $v(s)$ existe et elle est bornée dans l'intervalle $t \geqslant 0$. Il existe donc un $\lim v(s)=g$ quand $s \rightarrow \beta-0$, c'est-à-dire pour chaque $\varepsilon>0$ on a un intervalle $\left\langle s_{1}, \beta\right.$ ) Annales Polonici Mathematici XI 
tel que si $s \epsilon\left\langle s_{1}, \beta\right)$, alors $|v(s)-g|<\varepsilon$. A l'intervalle $\left\langle s_{1}, \beta\right)$ currespond, par la transformation inverse de la transformation $\left(7^{\prime}\right)$, l'intervalle $\left\langle t_{1}, \infty\right)$. Comme l'ensemble des valeurs $v(s)$ pour $s \epsilon\left\langle s_{1}, \beta\right)$ soit égal à l'ensemble des valeurs de l'intégrale correspondante $u(t)$ de l'équation différentielle (7) pour $t \epsilon\left\langle t_{1}, \infty\right)$, l'intégrale $u(t)$ tend vers une constante, quand $t \rightarrow+\infty$.

DÉfinition 1 . Nous dirons que la fonction $u(t) \not \equiv$ const est oscillante autour de $u=u_{0}$, quand $t \rightarrow a$, s'il existe une suite infinie de nombres $t_{n}$, $n=1,2, \ldots$ telle que $t_{n} \rightarrow \alpha$ et $u\left(t_{n}\right)=u_{0}$.

LEMare 1. La fonction $f(u)$, définie comme dans le théorème 2, doit changer de signe au moins une fois.

Démonstration. Si $f(u)$ ne changeait pas de signe pour $u \epsilon(-\infty$, $+\infty$ ), on n'aurait pas $F(u) \rightarrow+\infty$ quand $|u| \rightarrow+\infty$.

Défrnitron 2. Un zéro de la fonction $f(u)$, dans le voisinage duquel cette fonction change de signe, sera dit zéro essentiel de cette fonction.

LEMNE 2. Considérons l'équation différentielle (7), dans laquelle les fonctions $k(t)$ et $l(t)$ sont positives et continues dans l'intervalle $t \geqslant 0$. La fonction $f(u)$ satisfait aux mêmes conditions que dans le théorème 2 . Admettons que la fonction $u(t)$, satisfaisant aus conditions initiales données $u(0)=u_{0}$, $u^{\prime}(0)=u_{0}^{\prime}$, soit une intégrale de l'équation (7), définie et bornée dans l'intervalle $t \geqslant 0$.

Nous allons démontrer:

a) qu'il existe un $l$ tel que $\lim (u(t)-l)=0$ quand $t \rightarrow+\infty$, ou bien

b) que $\lim u(t) n^{\prime}$ existe pas pour $t \rightarrow+\infty$ et alors il existe un nombre $u^{*}$ qui est un zéro essentiel de la fonction $f(u)$ et la solution $u(t)$ oscille autour de $u=u^{*}$ pour $t \rightarrow \infty$. Si $\lim u(t)=l$ existe, quand $t \rightarrow+\infty$, et si le nombre $l$ $n$ 'est pas un zéro essentiel de la fonction $f(u)$, alors $u(t)$ tend vers $l$ d'une façon monotone, lorsque $t \rightarrow+\infty$.

Démonstration. En vertu des propriétés de transformation de $\left(7^{\prime}\right)$ il suffit, au lieu de la solution $u(t)$, de considérer une solution correspondante $v(s)$ de l'équation (8). Supposons que $\lim v(s)$ pour $s \rightarrow \beta-0$ n'existe pas. Alors $\limsup v(s)=M$ et $\liminf v(s)=m$ existent quand $s \rightarrow \beta-0$ et $M>m$. S'il existait un zéro essentiel $v_{1}$ de la fonction $f(v)$ tel que $m<v_{1}<M$, alors $v(s)$ oscillerait autour de $v=v_{1}$. Admettons que dans l'intervalle $(m, M)$ il n'existe pas de zéro essentiel de la fonction $f(v)$.

Nous allons démontrer que, dans ce cas, $m$ ou $M$ sont des zéros essentiels de la fonction $f(v)$. Dans le cas où ni $m$, ni $M$ ne serait pas un zéro essentiel de la fonction $f(v)$, il devrait exister des nombres $\varepsilon>0$ et $s_{1}<\beta$, tels que pour $s \in\left\langle s_{1}, \beta\right)$ on ait l'inégalité $m-\varepsilon<v(s)<M+\varepsilon$ et que l'intervalle $(m-\varepsilon, M+\varepsilon)$ ne contienne plus de zéros essentiels de la fonction $f(v)$. Il résulte de l'équation (8) que pour $s \epsilon\left\langle s_{1}, \beta\right)$ la dérivée $v^{\prime \prime}(s)$ ne change pas de signe, c'est-à-dire $v(s)$ est monotone, au moins à partir d'un $s_{2}\left(\beta>s_{2} \geqslant s_{1}\right)$. Donc, pour $\beta>s \geqslant s_{2}$, la fonction $v(s)$ est monotone et bornée. Il existe donc $\lim v(s)=l$ quand $s \rightarrow \beta-0$. II en résulte que le nombre $m$ ou $\mathbb{M}$ est un zéro essentiel de la fonction $f(v)$.

L'intégrale $v(s)$ doit osciller au moins autour d'une constante $v=m$ ou $v=M$, qui est un zéro essentiel de la fonction $f(v)$. Si $v(s)$ n'oscillait pas autour de $v=m$ ni $v=M, v^{\prime \prime}(s)$ ne changerait pas de signe, donc $v(s)$ devrait être monotone à partir d'un certain point.

$\mathrm{Du}$ dernier lemme et du théorème 2 résulte:

THÉOR亡̀me 7. Admettons que les fonctions $k(t), l(t)$ et $f(u)$ satisfassent à l'hypothèse $\mathrm{H}_{1}^{*}$. Dans ces conditions, l'ensemble des solutions de l'équation (7), définies pour $t \geqslant 0$, peut être divisé en deux classes $A$ et $B$, définies comme suit: $u(t) \in A$, s'il existe un nombre l tel que $\lim (u(t)-l)=0$, quand $t \rightarrow+\infty . u(t) \in B$, si $u(t) n^{\prime}$ appartient pas à la classe $A$. Il existe alors une constante $u=u^{*}$, qui est un zéro essentiel de la fonction $f(u)$, autour de laquelle oscille l'intégrale $u(t)$ quand $t \rightarrow+\infty$.

Corollatre 1. Considérons l'équation différentielle $u^{\prime \prime}+a(t) u^{\prime}+$ $+b(t) u=0$, dans laquelle la fonction $a(t) \epsilon C^{0}, b(t)>0$ et $b^{\prime}(t)$ est continue dans l'intervalle $\langle 0, \infty)$. En multipliant la dernière équation par $\exp \left(\int_{0}^{t} a(\tau) d \tau\right)$ nous obtenons

$$
\left(\exp \left(\int_{0}^{t} a(\tau) d \tau\right) u^{\prime}\right)^{\prime}+b(t) \exp \left(\int_{0}^{t} a(\tau) d \tau\right) u=0 .
$$

Si pour la fonction $k(t)=\exp \left(\int_{0}^{t} a(\tau) d \tau\right)>0$ et $b(t) \exp \left(\int_{0}^{t} a(\tau) d \tau\right)$ l'hypothèse H$_{1}^{*}$ est remplie, alors, en vertu du théorème 7 , toutes les solutions de l'équation (13) sont

$1^{\circ}$ bornées dans l'intervalle $t \geqslant 0$,

$2^{\circ}$ si les solutions de l'équation (13) sont non oscillantes dans l'intervalle $t \geqslant 0$, alors elles tendent vers des constantes d'une façon monotone, quand $t \rightarrow+\infty$.

On peut donner des exemples d'équations, telles que $u^{\prime \prime}-t u^{\prime}+$ $+\exp \left(t^{2}\right) u=0$, auxquelles on peut appliquer le corollaire cité ci-dessus, mais auxquelles ne s'applique pas le théorème donné par Z. Opial [11]. On peut aussi appliquer la dernière remarque aux équations $u^{\prime \prime}+t u^{\prime}+$ $+\exp \left(\frac{1}{2} t^{2}\right) u=0$ et $u^{\prime \prime}+(t-3)^{4}(t-1)^{-4} u=0$, auxquelles ne se rapportent pas les théorèmes, donnés par M. Matell [9] et généralisés par M. Zlámal ([16], th. 6 et th. 7 ).

Corollatre 2. En vertu du dernier corollaire on peut généraliser les théorèmes 3 et 4 , donnés par M. Ziámal ([16], p. 80 et 81). Notamment 
M. Zlámal démontre le théorème suivant: Si $\int^{\infty} a(s)^{-1} d s<+\infty$ et $a^{\prime}(t) a(t)^{-2}$ $\leqslant \delta<1$, alors l'équation

$$
u^{\prime \prime}+a(t) u^{\prime}+u=0,
$$

dans laquelle $a(t)>0$ pour $t \geqslant t_{0}$ possède une solution, tendant vers une constante différente de zéro. En vertu du corollaire 1 on peut obtenir, avec les mêmes hypothèses sur la fonction $a(t)$, que chaque solution $u(t)$ de l'équation (14) tende vers une constante d'une façon monotone, quand $t \rightarrow+\infty$.

Dans la même note, M. Zlámal démontre aussi le théorème suivant (p. 81): Si $a^{\prime}(t) \geqslant 1$ et $\int^{\infty}(a(s))^{-1} d s<\infty$, alors l'équation (14) possède une solution, tendant d'une façon monotone vers une constante, différente de zéro. On peut vérifier que pour le théorème 4, donné par M. Zlámal, les hypothèses du corollaire sont vraies. En vertu de ce fait chaque intégrale tend d'une façon monotone vers une constante, quand $t \rightarrow+\infty$.

Corolrame 3. Considérons l'équation différentielle

$$
\varepsilon(t) u^{\prime \prime}+u^{\prime}+u=0,
$$

dans laquelle $\varepsilon(t)>0$ et possède une dérivée $\varepsilon^{\prime}(t) \leqslant 2$ pour $t \geqslant t_{0}$. Comme dans le corollaire 1 , on peut montrer que les solutions de l'équation (15) sont bornées pour $t \geqslant t_{0}$. On connaît des résultats plus forts pour l'équation (15), quand $0<\varepsilon(t) \leqslant \frac{1}{2}$ (voir [16], th. 5, et [5]).

THस́oRk̀mE 8. Examinons l'équation différentielle (7), dans laquelle la fonction $k(t)$ est positive et continue pour $t \geqslant 0$ et $\int^{\infty} 1 / k(t) d t<+\infty$. Admettons que $\lim l(t)=g$ existe quand $t \rightarrow+\infty$ et $0<g<+\infty$. Si lè théorème 2 se rapporte d l'équation (12) et si la fonction $f(v)$ satisfait $\dot{a}$ la condition $f(v) v>0$ pour $v \neq 0$, alors la solution $u(t)$ de l'équation différentielle (7) est définie pour $t \geqslant t_{0}$, elle est oscillante et tend vers zéro, quand $t \rightarrow+\infty$.

Démonstration. Des hypothèses admises résultent les théorèmes 5 et 6, ce qui démontre la conclusion.

v. Nous passons maintenant à la démonstration de quelques théorèmes sur les solutions de l'équation différentielle

$$
u^{\prime \prime}(t)+a(t) \Phi(u(t)) \Phi_{u}^{\prime}(u(t))=0 \quad \text { pour } \quad t \geqslant t_{0}
$$

dont les suites des extêmes sont monotones.

HYPOTHÈsE $\mathbf{H}_{\mathbf{4}}$. La fonction $\Phi(u)$ appartient à la classe $C^{1}$. pour $u \in(-\infty,+\infty)$ et $\Phi(u) \Phi_{u}^{\prime}(u) u>0$ pour $u \neq 0$. La fonction $a(t)>0$ et il existe un $a^{\prime}(t)$, ne changeant pas de signe pour $t \geqslant t_{0}$.
CoRoluatre. La fonction $(\Phi(u))^{2}$ est décroissante pour $u \in(-\infty, 0)$ et croissante pour $u \in(0,+\infty)$.

THÉORÈme 9. Admettons que les fonctions $a(t)$ et $\Phi(u)$ satisfassent à l'hypothèse $\mathrm{H}_{4}$. Soit $u(t)$ une solution de l'équation différentielle (16), définie et oscillante pour $t \geqslant t_{0}$. Alors les suites des maxima et minima sont monotones. Plus précisément, si $a^{\prime}(t) \geqslant 0$ pour $t \geqslant t_{0}$, alors la suite des maxima est non croissante, et celle des minima est non décroissante, pour. $t \geqslant t_{0}$.

Mais si $a^{\prime}(t) \leqslant 0$ pour $t \geqslant t_{0}$, alors, inversement, la suite des maxima est non décroissante, et celle des minima est non oroissante pour $t \geqslant t_{0}$.

Si la fonction $\Phi(u) \Phi_{u}^{\prime}(u)$ était impaire, $\Phi(u) \Phi_{u}^{\prime}(u)=\Phi(-u) \Phi_{u}^{\prime}(-u)$, les modules des ectrêmes de l'intégrale $u(t)$ formeraient une suite monotone.

Démonstration. Considérons la fonction auxiliaire

On aura

$$
\varphi(t)=(\Phi(u(t)))^{2}+\left(u^{\prime}(t)\right)^{2} / a(t) .
$$

$$
\varphi^{\prime}(t)=-\frac{\left(u^{\prime}(t)\right)^{2}}{(a(t))^{2}} a^{\prime}(t) .
$$

Si $a^{\prime}(t) \geqslant 0$, alors $\varphi^{\prime}(t) \leqslant 0$ et $\varphi(t)$ n'est pas croissante pour $t \geqslant t_{0}$. Soient, par exemple, $t_{1}<t_{2}<\ldots$ les abscisses des maxima consécutifs de l'intégrale $u(t)$. On aura $\Phi\left(u\left(t_{i}\right)\right)>0$ et $u^{\prime}\left(t_{i}\right)=0$ pour $i=1,2, \ldots$ et la suite $\varphi\left(t_{i}\right)=\left(\Phi\left(u\left(t_{i}\right)\right)\right)^{2}$ est non croissante, d'où il résulte que la suite $u\left(t_{i}\right)$ est aussi non croissante. Les autres parties de la démonstration sont analogues.

Le dernier théorème reste en étroit rapport avec certains théorèmes, donnés dans [12], vol. II, chap. VII, § 4.

Remarque. Admettons que la fonction $l(t)=a(t)$ et $\Phi(u)$ satisfasse à l'hypothèse $H_{4}$. La fonction $k(t)>0$ est continue pour $t \geqslant t_{0}$ et $\int^{\infty} 1 / k(t) d t=+\infty$. Dans ces hypothèses les suites des maxima et des minima d'une solution arbitraire $u(t)$ de l'équation différentielle

$$
\left(k(t) u^{\prime}\right)^{\prime}+l(t) \Phi(u) \Phi_{u}^{\prime}(u)=0
$$

définie et oscillante pour $t \geqslant t_{0}$, sont monotones. Plus précisément: quand $l^{\prime}(t) \geqslant 0$ pour $t \geqslant t_{0}$, la suite des maxima est non croissante et celle des minima est non décroissante. Quand $l^{\prime}(t) \leqslant 0$, la suite des maxima est non décroissante et celle des minima est non croissante.

La démonstration résulte des propriétés de transformation de $\left(7^{\prime}\right)$.

\section{Travaux cités}

[1] R. Bellman, Stability theory of differential equation, New York 1953.

[2] Z. Butlewski, Sur les intégrales d'une équation différentielle du second ordre, Mathematica, Cluj, 12 (1936), p. 36-48. 
[3] L. Cesari, Asymptotic behavior and stability problems in ordinary differential equations, Berlin 1959.

[4] N. Coddington and N. Levinson, Theory of ordinary differential equations, New York 1955.

[5] T. Dłotko, 0 zachowaniu się calek równania rózniczkowego $a(t) u^{\prime \prime}+b(t) u^{\prime}+$ $+k^{a} u^{a}=\Phi(t)$, Zeszyty Nauk. W.S.P. w Katowicach, Nr 2 (1959), p. 11-18.

[6] - Quelques théorèmes sur les intégrales bornees de certaines équations différen. tielles ordinaires, Ann. Pol. Math. 10 (1961), p. 151-159.

[7] J. Kisyński, Remarque sur l'existence des solutions en large de l'équation $\partial^{2} z / \partial x \partial y=f(x, y, z, \partial z / \partial x, \partial z / \partial y)$, Ann. UMCS 13 (1959), p. 25-32.

[8] J. A. Klok off (Ю. А. Клоков), Некоторые теоремы об ограниченности реше ний обыкновенных дифферениияльных уравнений, Успехи Мат. Наук, Т. XIII, вып. 2 (80), 1958, p. 189-194.

[9] M. Matell, Asymptotische Eigenschaften gewisser linearer Differentialgleichungen, Uppsala 1924, Appelbergs Boktrykeri Aktiebolag.

[10] W. W. Niemyckij, W. W. Stiepanow (B. B. Немыцкий и B. В. Степа. нов), Качественная теория дифференцияльных уравнений, Москва 1949.

[11] Z. Opial, Sur l'allure asymptotique des intégrales de l'équation différentielle $u^{\prime \prime}+a(t) u^{\prime}+b(t) u=0$, Bull. Acad. Pol. Sci., Cl. III, 5 (1957), p. 847-853.

[12] G. Sansone, Equazioni Differenziali Nel Campo Reale, Parte Prima, Bologna 1948, Parte Seconda, Bologna 1949.

[13] W. W. Stiepanow (В. В. Степанов), Курс дифферениияльных уравнений, Москва 1953.

[14] T. Ważewski, Systèmes des équations et des inégalités différentielles ordinaires p. 112-167.

[15] A. Wintner, The nonlocal existence problem of ordinary differential equations, Amer. J. Math. 67 (1945), p. 277-284.

[16] M. Zlảmal, Über Asymptotische Eigenschaften der Lösungen der Linearen Differentialgleichungen zweiter Ordnung, Czechoslovak. Math. J. 6 (81) (1956), p. 75-91.

\section{Existence of Lyapunov functions}

by J. DuguNDJI * (Los Angeles, Calif.)

1. Introduction. We consider here the stability of the solutions of a non-autonomous system of differential equations $\dot{x}=X(x, t)$, where ( $\left.{ }^{1}\right)$ $X$ is continuous on $H \times J_{0}, H \subset E^{n}$ being a connected open set. There is no loss of generality to assume that the solution whose stability is being considered is $x=0$, so that $X(0, t) \equiv 0$. We moreover assume throughout this article: for each $\left(x_{0}, t_{0}\right) \in H \times J_{0}$ there exists a unique solution $x=x\left(t ; x_{0}, t_{0}\right)$ in $H$ which depends continuously on $\left(x_{0}, t_{0}\right)$, equals $x_{0}$ for $t=t_{0}$, and is defined for all $t \geqslant 0$; thus, we can take $\boldsymbol{H}=\boldsymbol{E}^{n}$. with no loss in generality.

The definition of uniform stability of $x=0$ can be stated in the following (normalized $\left({ }^{2}\right)$ ) way [1]: For each cylinder $C_{n} \equiv S(0,1 / n) \times$ $\times \dot{J}_{1-1 / n}, n=1,2, \ldots$, there exists a $C_{m}, m \geqslant n$, such that every trajectory entering $C_{m}$ remains thereafter in $C_{n}$; this type of stability is characterized by special properties of a Lyapunov function on $E^{n} \times J_{0}$, that is, a non-negative continuous real-valued function on $E^{n} \times J_{0}$ vanishing on $0 \times J_{1}$, bounded positively below outside each $C_{k}$, and having a continuous non-positive trajectory derivative $\left(^{3}\right)$ on $E^{n} \times J_{0}$. Now, if instead of cylinders, one is given a sequence $\left\{U_{n}\right\}$ of connected open sets in $E^{n} \times J_{0}$ with $0 \times J_{1}=\bigcap U_{n}$, then, replacing $U_{n}$ by $U_{n}$ in each of the above state-

* The author was partially supported by the National science Foundation, under contract G-5251, during the period that this work was done.

(1) Throuchout this article, $E^{n}$ denotes Euclidean $n$-space, and $J_{\alpha} \subset E^{1}$ the subspace $\{t \mid t \geqslant \alpha\}$. Vector notation is used. $S(x, \varepsilon)$ is the spherical neighborhood (nbd) of $x$ with radius $\varepsilon ; \dot{A}=$ boundary of $A ; A=$ interior of $A ; \circlearrowright A=$ complement of $A ;(x \bar{\epsilon} A)$ $\equiv\left(x \in e_{A}\right)$.

(2) The normalization consists in having the eylinders pinch down on $0 \times J_{1}$ rather than on some other $0 \times J_{a}$.

(3) For each $\left(x_{0}, t_{0}\right) \in E^{n} \times J_{0}$, the trajectory derivative $V^{\prime}\left(x_{0}, t_{0}\right)$ is the derivative $\frac{d}{d t} \nabla\left[x\left(t ; x_{0}, t_{n}\right), t\right]$ evaluated at $t=t_{0}$. We call a Lyapunow function proper (or simply "Lyapunov function") if $V^{\prime}\left(x_{0}, t_{0}\right)$ is continuous on $E^{n} \times J_{0}$; it is called "split" if $V^{\prime}\left(x_{0}, t_{0}\right)$ is continuous only on $e\left(0 \times J_{0}\right)$. 